PALEO

Revue d'archéologie préhistorique

$17 \mid 2005$

Varia

\title{
Note sur les talons en éperon du Protomagdalénien
}

Note on the Protomagdalenian spur-shaped butts

Frédéric Surmely et Philippe Alix

\section{(2) OpenEdition}

Journals

Édition électronique

URL : http://journals.openedition.org/paleo/908

DOI : $10.4000 /$ paleo.908

ISSN : 2101-0420

Éditeur

SAMRA

Édition imprimée

Date de publication : 1 décembre 2005

Pagination : 157-176

ISSN : 1145-3370

\section{Référence électronique}

Frédéric Surmely et Philippe Alix, « Note sur les talons en éperon du Protomagdalénien », PALEO [En

ligne], 17 | 2005, mis en ligne le 23 avril 2010, consulté le 07 juillet 2020. URL : http://

journals.openedition.org/paleo/908; DOI : https://doi.org/10.4000/paleo.908

Ce document a été généré automatiquement le 7 juillet 2020

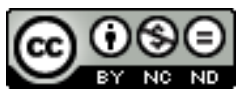

PALEO est mis à disposition selon les termes de la licence Creative Commons Attribution - Pas d'Utilisation Commerciale - Pas de Modification 4.0 International. 


\section{Note sur les talons en éperon du Protomagdalénien}

Note on the Protomagdalenian spur-shaped butts

Frédéric Surmely et Philippe Alix

M. Allard (Service régional de l'Archéologie de Midi-Pyrénées), J.-P. Bracco (ESEP-UMR 6636 Université de Provence), H. de Lumley et R. Nespoulet (Institut de Paléontologie Humaine), J.-J. Cleyet-Merle, Ph. Jugie et A. Morala (Musée national de Préhistoire des Eyzies-de-Tayac), J.-Ph. Rigaud (IPGQ, Bordeaux), J.-L. Rieu et J.-B. Roy (Musée de Préhistoire d'île-de-France), J. Pelegrin (C.N.R.S.), D. de Sonneville-Bordes, M. Olive (C.N.R.S.), B. Valentin (Université de Paris 1), C. Sestier et J. Tixier.

\section{Introduction}

1 Le Protomagdalénien est un faciès culturel encore peu connu. Caractérisé à LaugerieHaute, sur la base de deux critères, la présence de très grandes lames et d'une retouche scalariforme (Peyrony 1938), le Protomagdalénien (ou Périgordien VII) ${ }^{1}$, nous ne rentrerons pas ici dans le débat), se distingue aussi des autres technocomplexes du Paléolithique supérieur par sa rareté. Il n'a en effet été identifié clairement à ce jour que dans quatre gisements français : Laugerie-Haute-Est c. F, 36 et 38 (Les-Eyzies-deTayac, Dordogne ; Peyrony 1938 ; Bordes 1958 et 1978; Sonneville-Bordes 1960 ; Demars 1994), Abri Pataud c. 2 (Bosselin 1992 ; Clay 1995 ; Kong-Cho 1997), le Blot (Cerzat, Haute-Loire ; Delporte 1976; Bosselin 1992 et 1997) et les Peyrugues (Cabrerets, Lot; fouille M. Allard). Sa datation semble se placer autour de $22000 \mathrm{BP}^{2}$, d'après les datations ${ }^{14} \mathrm{C}$.

2 Les études concernant le Protomagdalénien ont toujours privilégié l'approche typologique. A l'occasion de la préparation de la publication du gisement du Blot (Cerzat, Haute-Loire) $)^{3}$, nous avons été amenés à reprendre les études sur le mobilier lithique des niveaux ${ }^{4}$ protomagdaléniens et à le considérer notamment sous l'angle technologique. Ceci nous a permis de constater l'existence de nombreux talons en 
éperon, ce qui n'avait pas été signalé par les chercheurs précédents. Ces aménagements particuliers, liés au débitage de grandes lames, étaient absents des séries des autres niveaux du gisement (Gravettien et Magdalénien moyen). Désireux de savoir si cette caractéristique se retrouvait dans d'autres sites du Protomagdalénien, nous avons alors entrepris une étude partielle des séries provenant des gisements de l'Abri Pataud et de Laugerie-Haute-Est, qui nous a permis de constater la présence de la même particularité et de confirmer la parenté étroite qui unit ces gisements. Enfin, ce travail a été complété par un examen très rapide de quelques séries du Magdalénien d'île-deFrance.

\section{Description}

Les éperons présents sur les talons des lames du Protomagdalénien sont aisément reconnaissables.

Certains sont massifs et proéminents, formant des excroissances bien marquées, à tel point qu'ils ont été parfois confondus avec des becs ou des fronts de grattoirs ogivaux (fig. 1 ; fig. $2 \mathrm{n}^{\circ} 1$ et 3 ; fig. $3 \mathrm{n}^{\circ} 1$, 5 et 6 ; fig. $5 \mathrm{n}^{\circ} 1$ et 3 ; fig. 6 ; fig. $7 \mathrm{n}^{\circ} 2$; fig. $8 \mathrm{n}^{\circ} 3$ et 4 ; fig. $9 \mathrm{n}^{\circ} 1$ et 3 ; fig. $10 \mathrm{n}^{\circ} 2$; fig. $11 \mathrm{n}^{\circ} 1$; fig. 12). Au Blot, la hauteur des éperons peut atteindre $5 \mathrm{~mm}$, pour une largeur de $10 \mathrm{~mm}$ (fig. $3 \mathrm{n}^{\circ} 5$; fig. 6). Ils ont été aménagés sur des plans de frappe très obliques, «déversés " (Inizan et al. 1995), avec des angles de chasse compris généralement entre 55 et $50^{\circ}$ et pouvant descendre jusqu'à $45^{\circ 5}$. Cette angulation particulière, conjuguée à la proéminence de l'éperon, a entraîné un véritable arrachement de la lame en arrière du point d'impact, donnant à la partie proximale de la lame une morphologie très caractéristique, avec une "lèvre " très marquée et une absence de bulbe de percussion (qui est même concave). Ce processus a été décrit avec précision par M. Brézillon (1971) et N. Pigeot (1987 et 2004), à propos des gisements des Tarterêts 2 et d'Étiolles. Au Blot, cet «arrachement » de lame a pu aller jusqu'au bris du support dans certains cas (fig. $2 n^{\circ} 3$; fig. $3 n^{\circ} 5$ et 6 ). Si au sein du groupe des lames à talons à éperon, les exemplaires à éperon massif constituent seulement $44 \%$ du nombre total, leur proportion s'élève à $70 \%$ pour ce qui est des pièces brisées au débitage. La cassure s'est faite dans une zone comprise entre 1 et $4 \mathrm{~cm}$ du talon (fig. $2 \mathrm{n}^{\circ} 3$ et fig. $3 \mathrm{n}^{\circ} 1$ et 6). Les fractures, envisagées sur le plan de la morphologie de leur surface appréhendée de profil (Ploux 1983), se partagent à proportions égales entre exemplaires à languette supérieure, languette inférieure et sans languette (cassure « franche ", Inizan et al. 1995) (fig. $3 \mathrm{n}^{\circ} 1$ et 6). 
Figure 1 - Le Blot. Protomagdalénien. Lames avec éperon massif. Figure 1 - Le Blot. Protomagdalenian. Blades with thick spurs.
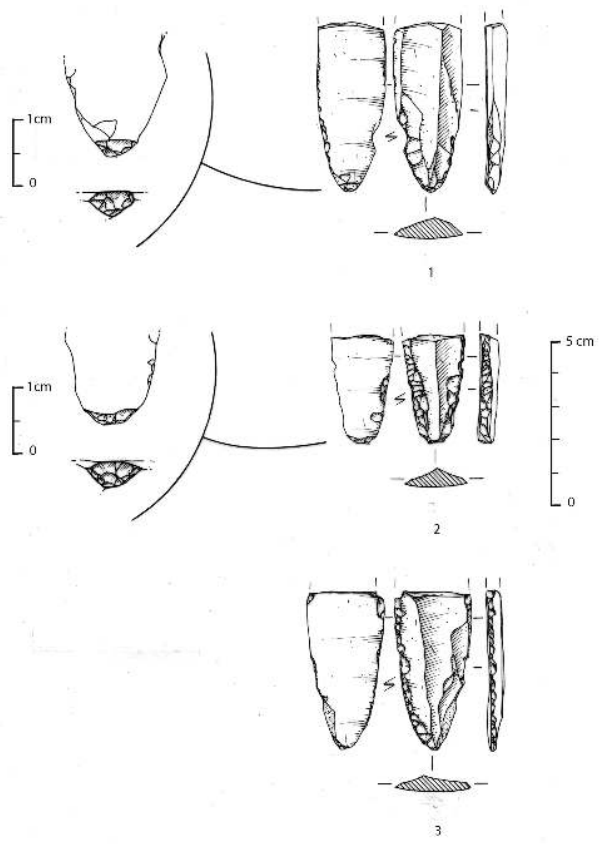

1 : silex pressignien ; 2 : silex d'origine inconnue ; 3 : silex de la craie

1 : pressignian flint ; 2 : flint of unknown origin ; 3 ; chalk flint.

Dessins: Ph. Alix.

Drawings : Ph. Alix. 
Figure 2 - Le Blot. Protomagdalénien. Figure 2 - Le Blot. Protomagdalenian.
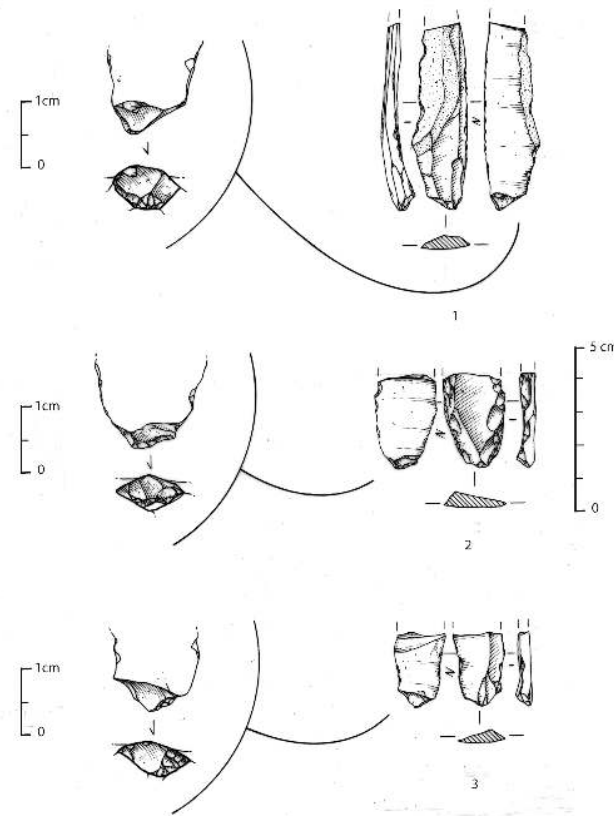

1 et 3 : lames avec éperon massif, en silex pressignien ; 2 : lame avec talon à facettage large, en silex de la craie.

1 and 3 : blades with thick spur, made out of pressinian flint : 2 : blade with broad-facetted butt, made out of chalk flint.

Dessins: Ph. Alix.

Drawings : Ph. Alix. 
Figure 3 - Le Blot. Protomagdalénien. Figure 3 - Le Blot - Protomagdalenian.
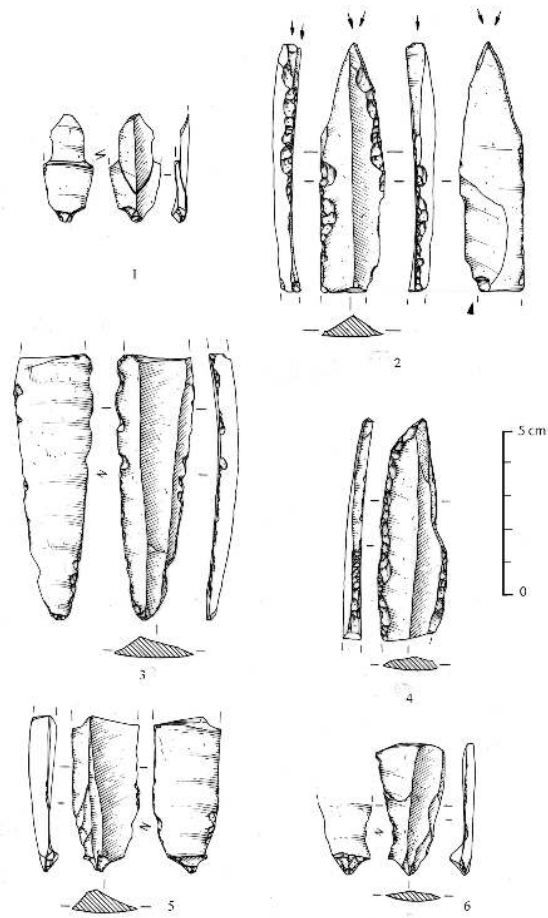

1,5 et 6 : lames à éperon massif ; le $n^{\circ} 1$ est un exemple de cassure en languette supérieure liée au débitage ; 3 : lame à éperon fin ; 2 et 4 : exemples de lames appointées, aménagées sur extrémité proximale du support (possédant probablement un éperon) et transformées ensuite en burins (sur troncature, puis dièdre).

7, 5 and $6:$ blades with thick spurs; $n^{\circ} 1$ is an example of upper " tongue-shaped » break due to the debitage ; 3 : blades with thin spurs; 2 and 4 : examples of pointed blades made on the proximal end of the blank (likely to have spurs) and which were later on turned into burins (truncation burins first, then dihedral ones).

Dessins: Ph. Alix

Drawings : Ph. Alix. 
Figure 4 - Le Blot. Protomagdalénien. Figure 4 - Le Blot. Protomagdalenian.

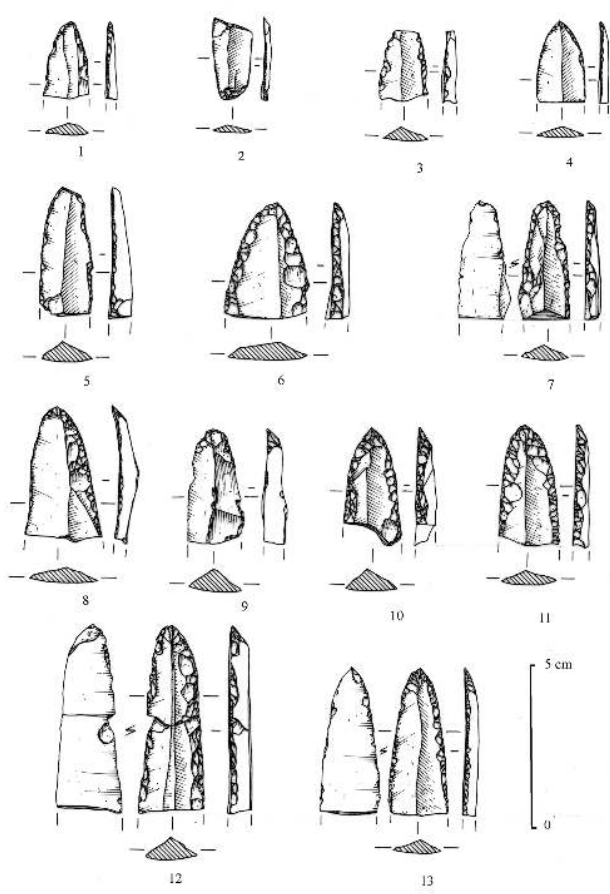

1 à 13 : lames appointées. Dans la très grande majorité des cas, l'appointement a été fait sur la partie proximale du support brut, ce qui a permis d'éliminer la saillie de l'éperon.

1 to 13 : pointed blades. In most cases, the blade was pointed on the proximal part of the untreated blank which made it possible to cut off the projecting part of the butt.

Dessins : Ph. Alix.

Drawings : Ph. Alix. 
Figure 5 - Le Blot. Protomagdalénien. Figure 5 - Le Blot. Protomagdalenian.

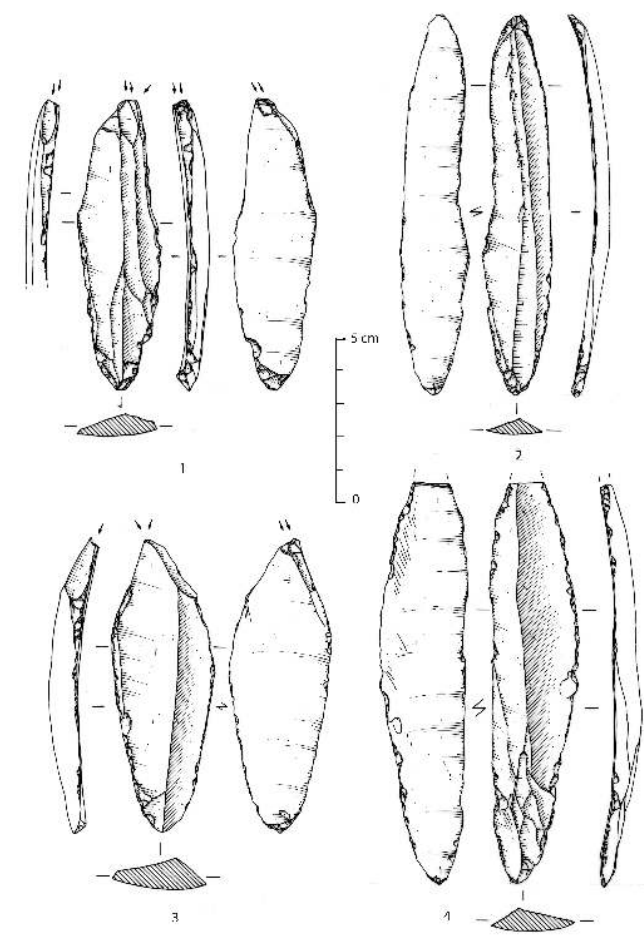

1 et 3 : éperons massifs ; 2 et 4 : éperons fins. Silex pressignien. 1 and 3 : thick spurs; 2 and 4 : thin spurs. Pressignian flint.

Dessins: Ph. Alix

Drawings : Ph. Alix. 
Figure 6 - Le Blot. Protomagdalénien. Figure 6 - Le Blot. Protomagdalenian.

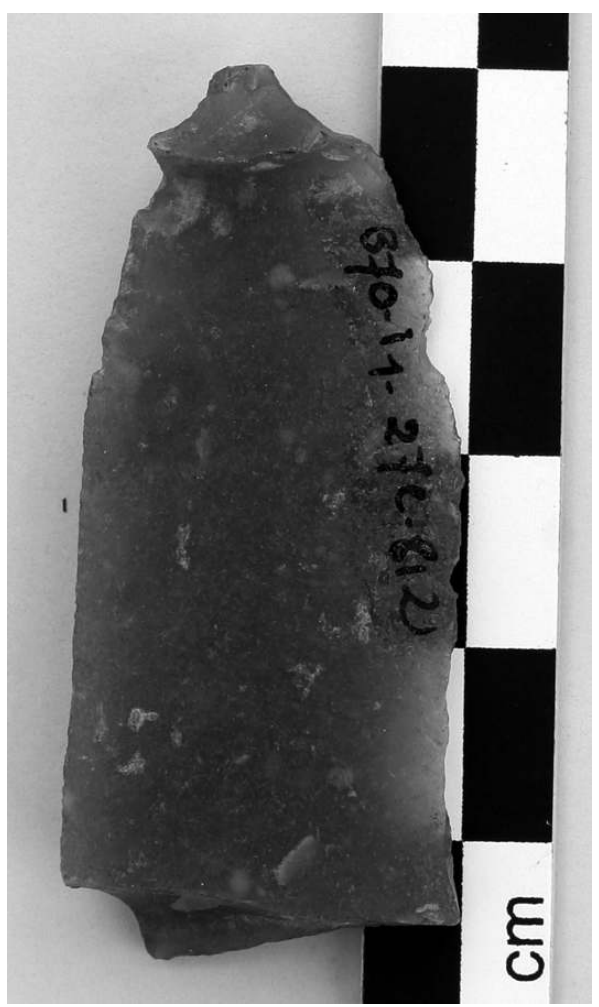

Photographie d'un éperon massif. Photography of a thick spur.

Photo Y. Duterne/SRA Auvergne.

Photo Y. Duterne/SRA Auvergne.

Il y a aussi des éperons plus fins et moins marqués (fig. $3 \mathrm{n}^{\circ} 3$; fig. $5 \mathrm{n}^{\circ} 2$ et 4 ; fig. 7 $n^{\circ} 1$; fig. $8 n^{\circ} 1$ et 2 ; fig. $9 n^{\circ} 2$ et 4 ; fig. $10 n^{\circ} 1$; fig. $11 n^{\circ} 2$ ), qui se rapprochent parfois des talons facettés (fig. $2 \mathrm{n}^{\circ}$ 2). Ils ont été aménagés par des enlèvements discrets qui peuvent avoir été faits avec l'aide d'un petit préparateur-abraseur. La proéminence de l'éperon est beaucoup plus discrète, avec des valeurs comprises entre 1 et $2,5 \mathrm{~mm}$, pour une largeur de 2 à $5 \mathrm{~mm}$. Sur ces pièces, la mesure de l'angle de chasse est impossible à réaliser. 
Figure 7 - Abri Pataud. Niveau 2. Figure 7 - Abri Pataud. Level 2.

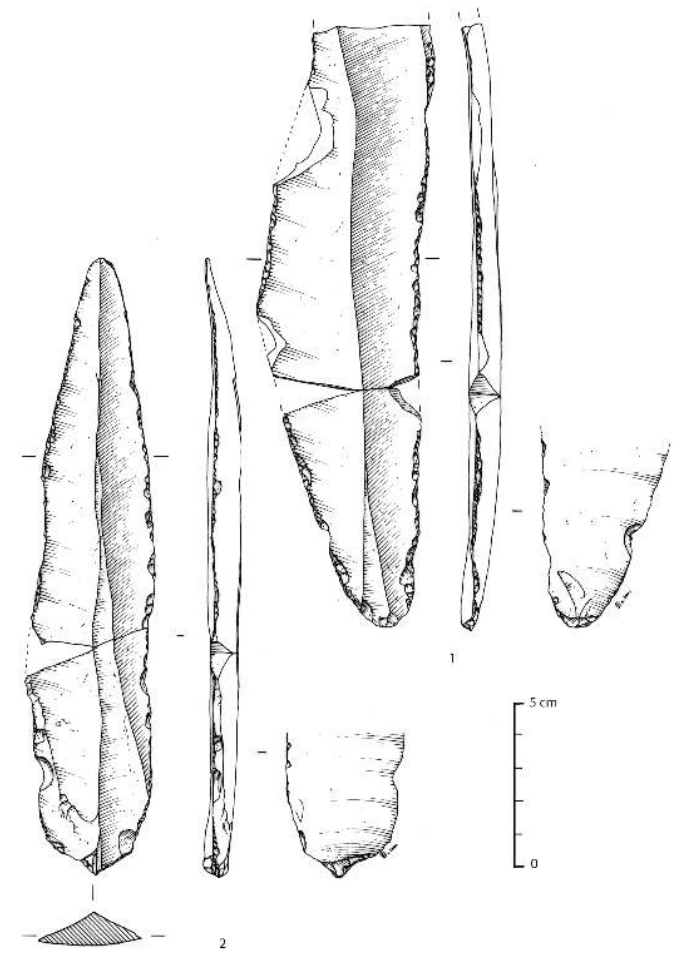

1 : éperon fin ; 2 : éperon massif. Silex du Bergeracois. 1 : thin spur ; 2 : thick spur. Flint from the Bergerac area. Dessins Ph. Alix. Drawings Ph. Alix. 
Figure 8 - Abri Pataud. Niveau 2.

Figure 8 - Abri Pataud. Level 2.

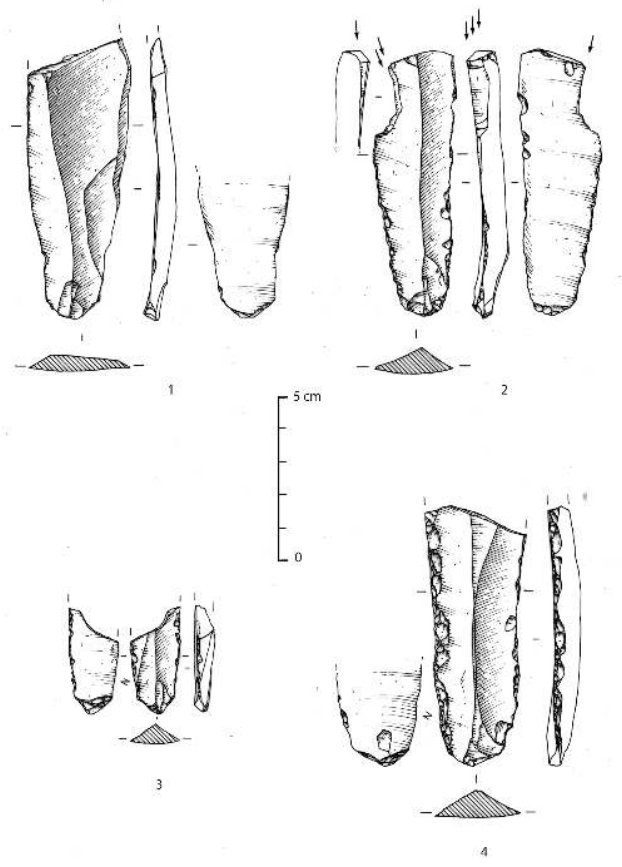

1 et 2 : éperons fins ; 3 et 4 : éperons massifs ; 3 : silex sénonien noir ; 4 : silex du Bergercois. 1 and 2 : thin spurs ; 3 and 4 : thick spurs ; 3 : black senonian flint ; 4 : flint from the Bergerac area. Dessins Ph. Alix.

Drawings Ph. Alix. 
Figure 9 - Abri Pataud. Niveau 2.

Figure 9 - Abri Pataud. Level 2.
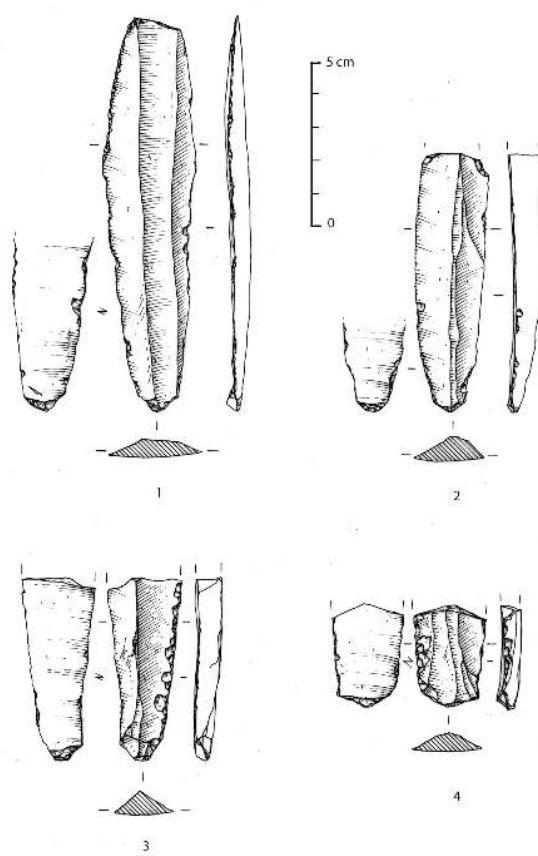

1 et 3 : éperons massifs ; 2 et 4 : éperons fins ; 1 à 3 : silex du Bergeracois ; 4 : silex sénonien noir 1 and 3 : thick spurs. 2 and 4 : thin spurs. 7 to 3 : flint from the Bergerac area. 4 black senonian flint Dessins Ph. Alix. Drawings Ph. Alix. 
Figure 10 - Abri Pataud. Niveau 2.

Figure 10 - Abri Pataud. Level 2.
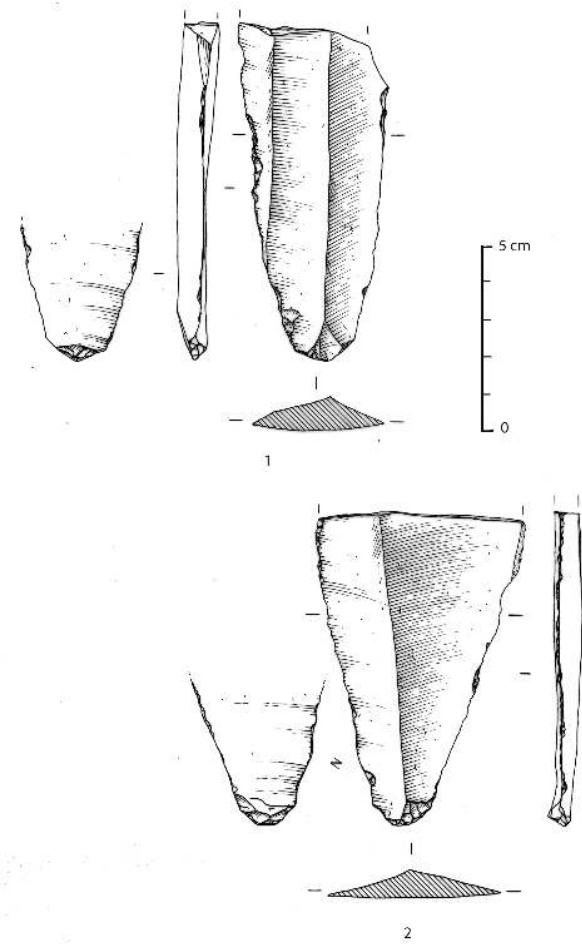

1 : éperon fin ; 2 : éperon massif. A noter que les deux éperons représentés sont de type intermédiaire

1 : thin spur ; 2 : thick spur. It should be noted that the two spurs shown are of an intermediate type.

Dessins Ph. Alix.

Drawings Ph. Alix. 
Figure 11 - Abri Pataud. Niveau 2.

Figure 11 - Abri Pataud. Level 2.

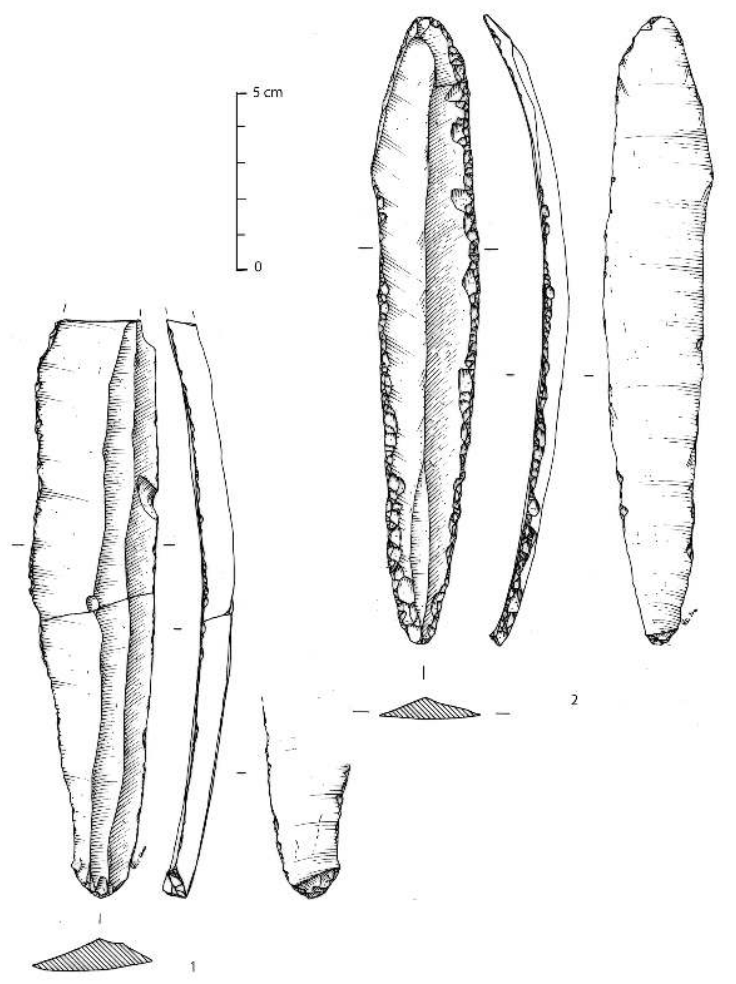

1 : éperon massif (silex coniacien blond) ; 2 : éperon fin (silex indéterminé)

1 : thick spur (blond coniacian flint) ; 2 : thin spur (indeterminable flint).

Dessins Ph. Alix.

Drawings Ph. Alix. 
Figure 12 - Laugerie-Haute-Est. Niveau 36. Figure 12 - Laugerie-Haute-Est. Level 36.

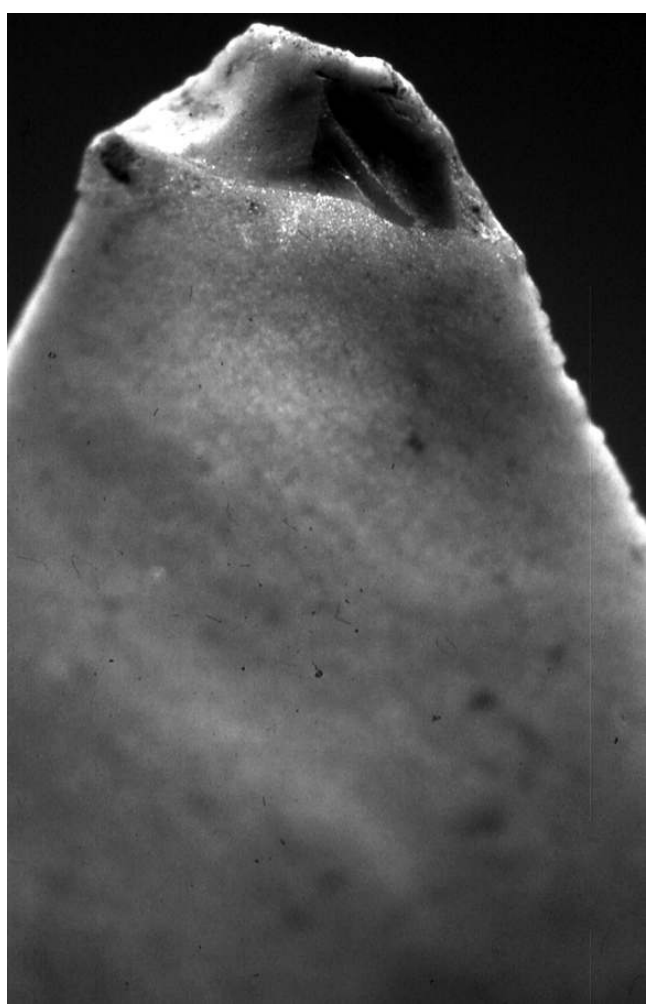

Éperon massif

Thick spur.

Photo Ph. Jugie/MNP.

Photo Ph. Jugie/MNP. 
Figure 13 - Le Blot. Protomagdalénien.

Figure 13 - Le Blot. Protomagdalenian.

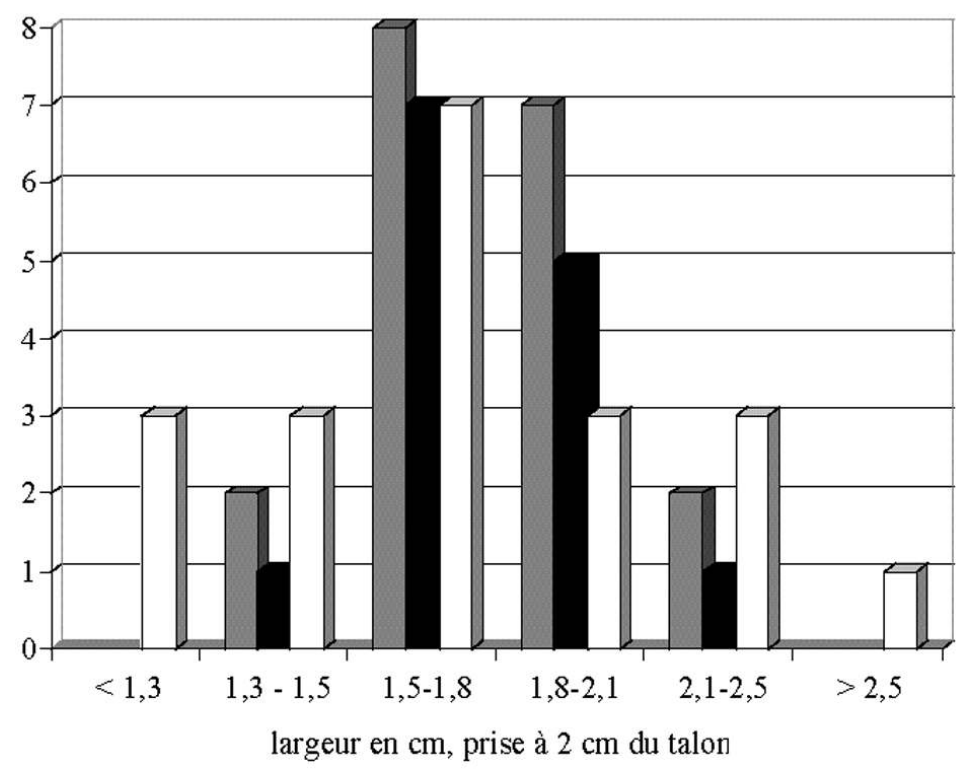
$\square$ EP. fin
EP. massif

$\square$ Autres

Répartition des types de talons par classe de largeur des lames (mesure prise à $2 \mathrm{~cm}$ de l'extrémité proximale).

Distribution of butt types according to the width of the blades (measured $2 \mathrm{~cm}$ from the proximal end).

Dans le cadre de l'étude, nous avons cru utile de séparer les exemplaires «massifs » des exemplaires "fins ", afin d'isoler d'éventuelles spécificités. Cela dit, il n'existe pas de limite morphologique nette entre les exemplaires « massifs » et les exemplaires « fins ». De nombreux éperons sont ainsi à la limite entre les deux « types » (fig. $9 \mathrm{n}^{\circ} 4$; fig. 10).

Malgré la présence d'un supposé chasse-lame signalé par F. Bordes dans les séries de Laugerie-Haute ${ }^{6}$ (Bordes 1978), la technique utilisée est probablement la percussion directe au percuteur tendre (Inizan et al. 1995 ; Pigeot 1987 ; Gallet 1998).

Les Protomagdaléniens semblent avoir cherché à faire disparaître la saillie gênante (notamment pour l'emmanchement) que formait le talon en éperon, notamment quand celui-ci était marqué. Le moyen le plus fréquemment utilisé était celui de l'aménagement d'une lame appointée (parfois appelée " grattoir ogival », voire " pièce à retouches continues", selon les auteurs), elle-même servant souvent de base pour la fabrication d'un burin (fig. $3 \mathrm{n}^{\circ} 2$ et 4 ; fig. 4). On observe en effet que l'appointement des lames a été fait en très forte majorité sur la partie proximale des supports (fig. 4 et tabl. 1). Ce phénomène se retrouve à l'identique sur les trois gisements protomagdaléniens examinés (par exemple: Bordes 1978, fig. $5 \mathrm{n}^{\circ} 4$ et fig. $10 \mathrm{n}^{\circ} 13$ ). Nous décrirons ailleurs ce processus qui pourrait éclairer d'un jour nouveau l'approche typologique de ces pièces. Cette élimination quasi systématique des talons explique qu'ils soient rarement conservés. Les éperons "subsistants" se retrouvent majoritairement (10 éperons massifs sur un total de 15, au Blot) sur des produits laminaires brisés au cours du débitage et non utilisés (fig. $2 \mathrm{n}^{\circ} 3$ et fig. $3 \mathrm{n}^{\circ} 1$ et 6 ). 
Tableau 1 - Le Blot et Laugerie-Haute-Est (fouilles Peyrony et Bordes).

Table 1 - Le Blot and Laugerie-Haute-Est (excavated by Peyrony and Bordes).

\begin{tabular}{|l|l|l|l|}
\hline Extrêmité appointée (en \%) & Proximale & Distale & Proximale et distale \\
\hline $\begin{array}{l}\text { Le Blot } \\
(\mathrm{n}=30)\end{array}$ & 70 & 27 & 3 \\
\hline $\begin{array}{l}\text { Laugerie-Haute-Est } \\
\text { niv. 36 et } 38 \\
(\mathrm{n}=34)\end{array}$ & 68 & 23 & 9 \\
\hline
\end{tabular}

Niveaux protomagdaléniens. Localisation des appointements sur les lames.

Protomagdalenian levels. Location of the pointing on the blades.

\section{Fréquence des talons en éperon}

9 Il est difficile de connaitre leur fréquence exacte, compte tenu de leur élimination ultérieure dans de très nombreux cas, avant utilisation du support, comme nous l'avons dit plus haut. Ainsi, au Blot, seuls 69 talons sont conservés, sur un total de 391 supports laminaires. Cela constitue bien sûr une gêne pour l'étude, notamment pour les calculs statistiques. Les éperons sont présents sur 12 à $51 \%$ des talons conservés ${ }^{7}$, selon les gisements et les niveaux (tabl. 5). Ce pourcentage est assurément inférieur à la réalité initiale.

Les éperons concernent tous les types de silex. Nous avions déjà fait la même remarque, pour les seuls éperons fins, à propos du site du Magdalénien final du Pont-de-Longues (Surmely et al. 2002). Sur le site du Blot, les deux types ont été aménagés indifféremment sur les deux principales matières premières utilisées par les tailleurs (tabl. 4) : le silex de la craie du Berry et celui du Grand-Pressigny, qui ont pourtant des caractéristiques différentes (Surmely et Murat 2003). Le silex de la craie a une texture plus fine et plus homogène, tout en offrant des modules initiaux plus petits. De même, à Pataud, l'aménagement d'éperons massifs concerne tout aussi bien le silex bergeracois importé que les silex crétacés locaux (tabl.2). A Laugerie, les éperons sont surtout observables sur le silex du Bergeracois, ce qui s'explique par le module généralement supérieur des produits laminaires (tabl. 3).

Tableau 2 - Abri Pataud. Niveau 2.

Table 2 - Abri Pataud. Level 2.

\begin{tabular}{|l|l|l|l|l|l|l|l|l|l|}
\hline Cortical & Lisse & $\begin{array}{l}\text { Lisse } \\
\text { abrasé }\end{array}$ & Dièdre & Linéaire & $\begin{array}{l}\text { Eperon } \\
\text { fin }\end{array}$ & $\begin{array}{l}\text { Eperon } \\
\text { massif }\end{array}$ & Facetté & $\begin{array}{l}\text { Puncti- } \\
\text { forme }\end{array}$ \\
\hline $\begin{array}{l}\text { Lames } \\
\text { brutes } \\
(\mathrm{n}=99)\end{array}$ & & 1 & 31 & 1 & 52 & 3 & 1 & 4 & 6 \\
\hline
\end{tabular}




\begin{tabular}{|l|l|l|l|l|l|l|l|l|l|}
\hline $\begin{array}{l}\text { Pièces } \\
\text { utilisées } \\
(\mathrm{n}=90)\end{array}$ & 5 & 26 & 4 & 43 & 3 & 1 & 2 & 6 \\
\hline $\begin{array}{l}\text { Outils } \\
(\mathrm{n}=82)\end{array}$ & & 2 & 25 & 2 & 16 & 16 & 9 & 9 & 3 \\
\hline $\begin{array}{l}\text { Total } \\
(\mathbf{e n} \%) \\
(\mathbf{n}=\mathbf{2 7 1})\end{array}$ & $\mathbf{3}$ & $\mathbf{3 0}$ & $\mathbf{2 , 5}$ & $\mathbf{4 1}$ & $\mathbf{8}$ & $\mathbf{4}$ & $\mathbf{5 , 5}$ & $\mathbf{5 , 5}$ \\
\hline
\end{tabular}

Talons des lames, tous types de matières premières confondus. Blade butts, from all sorts of raw materials.

Tableau 3 - Laugerie-Haute-Est (fouilles F. Bordes). Niveau 36. Table 3 - Laugerie-Haute-Est (excavated by F. Bordes). Level 36.

\begin{tabular}{|l|l|l|l|l|l|l|l|l|l|}
\hline & Cortical & Lisse & $\begin{array}{l}\text { Lisse } \\
\text { abrasé }\end{array}$ & Dièdre & Linéaire & $\begin{array}{l}\text { Eperon } \\
\text { fin }\end{array}$ & $\begin{array}{l}\text { Eperon } \\
\text { massif }\end{array}$ & Facetté & $\begin{array}{l}\text { Puncti- } \\
\text { forme }\end{array}$ \\
\hline $\begin{array}{l}\text { Lames } \\
\text { brutes } \\
(\mathrm{n}=124)\end{array}$ & 2 & 33 & 3 & 44 & 12 & 9 & 14 & 7 \\
\hline $\begin{array}{l}\text { Pièces } \\
\text { utilisées } \\
(\mathrm{n}=13)\end{array}$ & 1 & & 3 & & 2 & 3 & 3 & 2 & \\
\hline $\begin{array}{l}\text { Outils } \\
(\mathrm{n}=33)\end{array}$ & 1 & & 7 & & 7 & 7 & 5 & 4 & 2 \\
\hline $\begin{array}{l}\text { Total } \\
(\mathbf{e n} \%) \\
(\mathbf{n}=\mathbf{1 7 0})\end{array}$ & $\mathbf{0 , 5}$ & $\mathbf{1}$ & $\mathbf{2 5}$ & $\mathbf{2}$ & $\mathbf{3 1}$ & $\mathbf{1 3}$ & $\mathbf{1 0}$ & $\mathbf{1 2}$ & 5,5 \\
\hline
\end{tabular}

Talons des lames (sélection), tous types de matières premières confondus. Blade butts (selection), from all sorts of raw materials.

Il ne semble donc pas y avoir de corrélation entre éperon et type de silex.

11 En revanche, les talons en éperon se retrouvent exclusivement sur les lames et surtout sur les beaux produits de plein débitage, ce qui explique qu'ils soient surtout associés aux outils (tabl. 3 et 4 ) et bien calibrés en largeur (fig. 13). Ils se retrouvent aussi sur des lames à crête ou d'entame. Ils sont totalement absents sur les éclats. Cela confirme bien le lien étroit qui les unit au débitage laminaire, mis en évidence sur les gisements magdaléniens d'île-de-France (Pigeot 1987 et 2004 ; Pelegrin 1992 ; Inizan et al. 1995).

12 Il semble donc exister un lien fort entre la dimension des supports laminaires et l'aménagement d'éperons, ces derniers étant plus fréquents sur les lames de grand module. Cette observation a pu être faite également sur les gisements des Tarterêts I (Karlin 1975) et d'Étiolles (Pigeot 1987 et 2004). 

composé de supports laminaires fragmentés, parfois réduits à une longueur d'un ou deux centimètres (les lames entières ayant conservé leur talon intact sont rares ; 11 exemplaires recensés !). La relation directe entre éperon et longueur de lame est donc impossible à établir. En prenant le critère de la largeur (fig. 13), aucune évidence n'apparaît non plus, mais il est vrai que la mesure de la largeur à $2 \mathrm{~cm}$ du talon, rendue nécessaire, n'est pas forcément significative de la largeur maximale du support. Notons toutefois que sur le gisement magdalénien de Marolles, les éperons se trouvent en majorité sur les lames larges (Valentin et al. 1999). De même, la faiblesse statistique de l'échantillonnage du Blot empêche de comparer la représentation des types d'éperons en fonction de la courbure du profil de la lame.

montrent que l'épaisseur des supports à talon en éperon est généralement supérieure à celle des lames ayant un autre type de talon (hors talon facetté). De même, les lames à talon en éperon sont mieux calibrées en largeur (fig. 13) que les autres, comme nous l'avons dit plus haut.

\section{Fonction et impact sur la consommation de matière première}

La fonction des éperons, décrite sur le gisement d'Étiolles (Pigeot 2004), correspond à la volonté de renforcer le plan de frappe pour réduire sensiblement le risque d'éclatement ou d'esquillement de celui-ci et de guider dans les deux sens l'impact du percuteur. Ceci facilite sensiblement le débitage de lames de grande taille. Cette préparation du point de percussion est particulièrement utile pour les grandes et larges lames peu arquées, qui ont un large talon et dont le débitage nécessite une percussion plus puissante. Ceci est bien visible sur les larges lames en silex du Bergeracois retrouvées sur les sites de l'abri Pataud et de Laugerie-Haute.

16 Il convient toutefois de signaler que le procédé de l'éperon n'est pas obligatoire pour le détachement de lames, y compris de celles de grandes dimensions. Ainsi au Blot, une part non négligeable des grands supports n'a pas reçu ce type d'aménagement et les talons sont alors de type lisse abrasé (fig. 13). De ce fait, l'aménagement en éperon est considéré comme ayant une « charge culturelle très forte » (Pigeot 2004).

L'incidence du choix de l'aménagement d'éperons sur l'économie de la matière première est plus difficile à mesurer. Cette question est d'autant plus importante qu'une part notable, voire presque exclusive dans le cas du Blot (Surmely et Pasty 2003 ; Surmely et al. soumis), de la matière première utilisée dans les gisements protomagdaléniens considérés était d'origine lointaine. Cette gestion rigoureuse s'observe très nettement au Blot, où $90 \%$ des matériaux proviennent de plus de $250 \mathrm{~km}$, notamment au travers des ravivages multiples des outils et de l'emport des nucléus lors de l'abandon du site.

18 Tous les chercheurs ayant étudié le sujet (Pelegrin 1992 ; Valentin et al. 1999 ; Pigeot 2004) ont souligné que le procédé de l'éperon est «techniquement et économiquement coûteux » (Pigeot 2004), puisqu'il nécessite notamment des ravivages assez fréquents du plan de frappe. Ceci se voit bien sur les remontages de tablettes et éclats de ravivage, comme à l'abri Pataud. Toutefois, ce dernier auteur fait remarquer que la fréquence des

PALEO, 17 | 2005 
ravivages du plan de frappe est limitée dans le cas de plans de frappe très obliques, qui ont été privilégiés effectivement par les Protomagdaléniens, notamment au Blot. Les tailleurs ont parfois détaché de simples petits éclats de ravivage, surcreusant le plan de frappe (fig. 14) et se limitant ainsi à une obliquité partielle, moins dispendieuse à court terme, mais nécessitant ensuite l'enlèvement d'une tablette assez épaisse. L'incidence de l'utilisation de l'éperon sur la fréquence de fracturation des lames est difficile à estimer. Si l'on observe des cas de fragilisation de la partie antérieure du support, voire de sa rupture (cf. supra), de l'autre côté, l'éperon en assurant un contact préférentiel et prédéterminé de l'impact du percuteur, apporte un surcroît de sécurité dans le débitage. Il n'en reste pas moins qu'une part parfois importante des lames semble s'être brisée lors du détachement. Ceci reste à préciser par des études complémentaires.

Figure 14 - Les Tarterêts.

Figure 14 - Les Tarterêts.

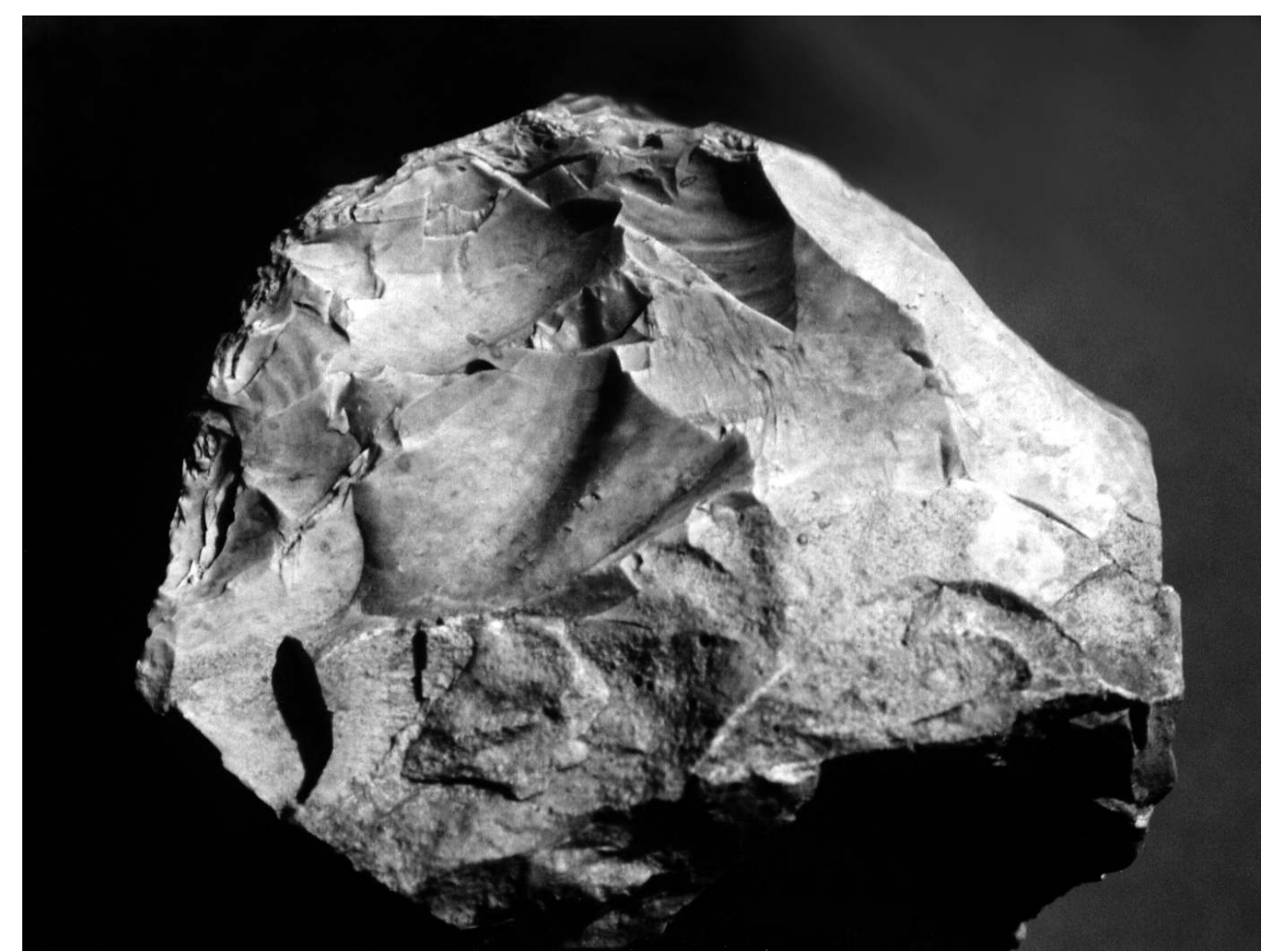

Vue supérieure d'un nucléus à lames montrant le surcreusement partiel du plan de frappe, du côté de la table, lié à l'aménagement d'éperons.

A blade seen from above showing how the striking platform was partially hollowed,due to the making of spurs.

Photo J.-L. Rieu/MPIF.

Photo J.-L. Rieu/MPIF.

19 L'utilisation très fréquente du procédé de l'éperon sur les sites protomagdaléniens où la matière première était d'origine lointaine montre nettement que cette pratique, si elle ne paraissait pas économique au premier abord, s'avérait «correspondre à un choix manifeste d'optimisation du débitage laminaire » (Valentin et al. 1999).

En résumé, on peut penser que le surcoût d'investissement technique et économique lié à l'aménagement d'éperon a été compensé par la garantie plus grande de rentabiliser le débitage laminaire, notamment dans le cas de la production de supports de grande taille. 


\section{Comparaison avec d'autres cultures}

21 Les éperons sont connus depuis l'Aurignacien (Inizan et al. 1995 ; com. pers. J. Tixier) et liés au débitage laminaire. Les éperons fins sont assez fréquents dans l'industrie lithique des phases finales du Magdalénien, notamment sur les sites du Bassin Parisien (Audouze et al. 1988), comme par exemple Étiolles (Pigeot 1987 et 1988), Pincevent (Karlin 1972), Les Tarterêts (Brézillon 1971 ; Karlin 1975 ; Schmider 1975 ; Florès 1998), Marsangy (Pelegrin 1992), Marolles-sur-Seine (Valentin et al. 1999) et Les Gros Monts (Cheynier 1957), ou bien d'Auvergne (Bérard 1995 ; Séara 1998 ; Surmely 1998 ; Surmely et al. 2002). Il sont également présents dans le Creswellien, équivalent anglais du Magdalénien (Barton et Dumont 2000). Toutefois, ces éperons sont très souvent de petites dimensions, proches du simple facettage. C'est le cas, dans le Bassin parisien, de Pincevent, de Marolles (Valentin et al. 1999 ; com pers. J.-.L Rieu), mais aussi des GrosMonts 8

Les éperons massifs sont en revanche beaucoup moins fréquents, à notre connaissance. Ils sont très faiblement représentés dans le Gravettien ancien du gisement du Sire (Mirefleurs, Puy-de-Dôme; Surmely, inédit) et n'apparaissent pas dans les niveaux du Gravettien moyen et final, notamment dans les sites concernés dans la présente étude : Le Blot (Klaric 1999), Abri Pataud (Nespoulet 1996 et com. pers.), Laugerie-Haute-Est. Ainsi, sur ce dernier site, ils sont quasiment absents dans le niveau du Périgordien VI (niveau D, fouilles Peyrony) qui est directement sous-jacent aux couches protomagdaléniennes et où les produits laminaires sont en général de taille inférieure à ceux découverts dans le Protomagdalénien (tabl.4). Ils se retrouvent toutefois dans quelques sites de la région de Bergerac, où ils sont associés à un débitage laminaire de très grande taille. Ils ont été ainsi observés sur les sites de Canaule I et Troche (Creysse, Dordogne), dans un contexte chronologique mal déterminé (Guichard et Morala 1989). Ils sont fréquents dans l'industrie du gisement de Rabier qui est daté de la fin du Gravettien ou du Protomagdalénien (Périgordien VI ou VII) (Morala 1990 ; Lorin 1996 ; com. orale A. Morala) et de Corbiac (com. pers. J. Pelegrin). Toutefois les éperons de Rabier, bien que très massifs, sont rarement aussi proéminents que ceux observés dans le Protomagdalénien. Nous n'avons pas de données concernant le Solutréen.

Tableau 4 - Le Blot - Protomagdalénien (tous niveaux).

Table 4 - Le Blot - Protmagdalenian (all levels).

\begin{tabular}{|l|l|l|l|l|l|l|l|l|l|}
\hline & Cortical & Lisse & $\begin{array}{l}\text { Lisse } \\
\text { abrasé }\end{array}$ & Dièdre & Linéaire & $\begin{array}{l}\text { Eperon } \\
\text { fin }\end{array}$ & $\begin{array}{l}\text { Eperon } \\
\text { massif }\end{array}$ & Facetté & $\begin{array}{l}\text { Puncti- } \\
\text { forme }\end{array}$ \\
\hline $\begin{array}{l}\text { Silex Gd } \\
\text { Pressigny } \\
(\mathrm{n}=32)\end{array}$ & & 1 & 2 & 1 & 7 & 11 & 7 & 2 & 1 \\
\hline $\begin{array}{l}\text { Silex de la } \\
\text { craie } \\
(\mathrm{n}=36)\end{array}$ & & 1 & 5 & & 7 & 8 & 8 & 4 & 3 \\
\hline
\end{tabular}




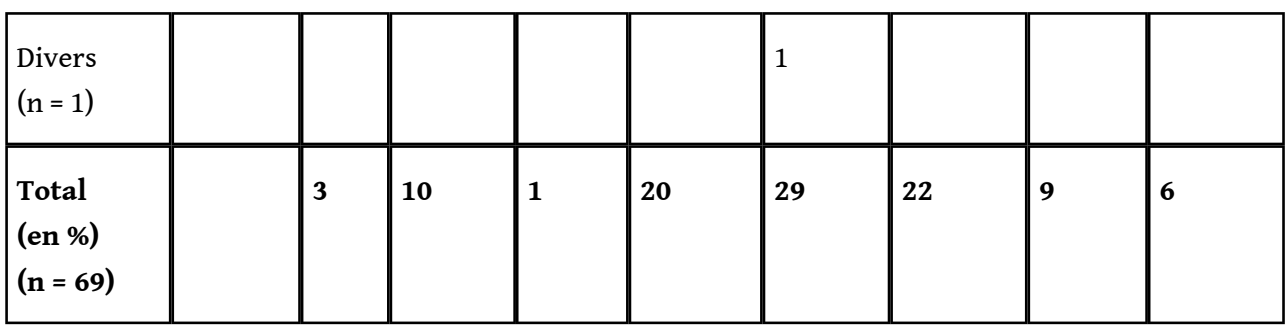

Talons des supports laminaires, bruts ou transformés, selon les types de matières premières. Blade butts, either untreated or treated, according to the types of raw materials.

Tableau 5 - Proportion des talons en éperon (en \% pour chaque niveau) pour les lames brutes et transformées.

Table 5 - Ratio of spur-shaped butts (percentage for each level) for untreated and treated blades.

\begin{tabular}{|l|l|}
\hline Gisements étudiés & $\%$ des talons en éperon \\
\hline Laugerie-Haute-Est - Protomagdalénien - Fouilles Bordes - niv. 36 & 23 \\
\hline Laugerie-Haute-Est - Protomagdalénien - Fouilles Bordes - niv. 38 & 46 \\
\hline Laugerie-Haute-Est - Protomagdalénien - Fouilles Peyrony - niv. F & 35 \\
\hline Pataud - Protomagdalénien - niv. 2 & 12 \\
\hline Le Blot - Protomagdalénien - tous niveaux & 51 \\
\hline Laugerie-Haute-Est - Périgordien VI - Fouilles Peyrony - niv. D & 6 \\
\hline
\end{tabular}

A notre connaissance, les seuls spécimens comparables, d'un point de vue qualitatif et quantitatif, à ceux du Protomagdalénien ne se rencontrent que dans quelques gisements du Magdalénien d'île-de-France, comme Étiolles et Les Tarterêts, où le débitage laminaire est tourné vers la production de grandes lames. Aux Tarterêts 1, les talons en éperon sont représentés sur $95 \%$ de l'échantillonnage de lames que nous avons pu examiner, avec un rapport de $60 / 40 \%$ en faveur des éperons de type massif. Le procédé se retrouve bien sûr sur les nucléus (fig. 14). Cette proportion étonnante d'éperons conservés est à mettre en relation avec la très importante fracturation des supports lors du débitage. Les éperons sont également très présents dans le plein débitage d'Étiolles (Pigeot 2004), avec des formes massives en tous points comparables aux talons du Protomagdalénien. L'éperon figurant sur la lame T1043 fait ainsi $9 \mathrm{~mm}$ de hauteur ${ }^{9}$ (Olive 1988). Les éperons sont nettement moins fréquents et moins proéminents sur les lames du site des Tarterêts 2 , où ils sont souvent proches du simple facettage.

\section{Conclusion}

Les tailleurs du Protomagdalénien, qui cherchaient à produire de grandes lames régulières, ont eu recours, de façon très fréquente, à l'aménagement d'éperons. 
A côté des éperons fins se trouvent des exemplaires massifs et très proéminents, qui ont donné une forme particulière à la partie proximale des supports. Certainement gênants pour l'utilisation ultérieure, ils ont souvent été éliminés par la suite, essentiellement par retouche. L'aménagement de ces éperons massifs, que l'on retrouve à l'identique sur les séries des trois gisements étudiés, semble donc bien caractéristique du débitage laminaire du Protomagdalénien, axé sur la recherche de lames de grandes dimensions, à partir de silex souvent importés. Ce type de préparation se retrouve dans les séries de grandes lames de certains sites du Magdalénien final d'île-de-France, comme Étiolles, ce qui confirme le lien étroit qui existe entre éperons et recherche d'un débitage laminaire de grande taille. Mais comme le souligne N. Pigeot, l'aménagement d'éperons va probablement au-delà d'un simple geste (Pigeot 2004). En revanche, sur les sites concernés (Laugerie, Pataud, Le Blot), ces aménagements sont absents des niveaux d'occupation gravettiens antérieurs au Protomagdalénien, ce qui va dans le sens d'une individualité de cette dernière culture par rapport au complexe gravettien.

\section{BIBLIOGRAPHIE}

Audouze F., KARLin C., CAHEN D., CROISSET E. de, COUdRET P., LARRIÈRE M., MASSON P., MAUGER M., OLIVE M., PÉLEGRIN J., PIGEOT N., PLISSON H., SCHMIDER B. et TABORIN Y. 1988 - Taille du silex et finalité du débitage dans le Magdalénien du Bassin Parisien. In Otte M. (ed.) : De la Loire à l'Oder. Les civilisations du paléolithique final dans le nord-ouest européen, BAR, 444, p. 55-84.

BARTON N. et DUMONT S. 2000 - Recolonisation and settlement of Britain at the end of the last glaciation. In : L'Europe centrale et septentrionale au tardiglaciaire, Table ronde de Nemours, mai 1997, Mémoires du Musée de Préhistoire d'île de France, 7, Nemours, édition de l'APRAIF, p. 151-162. BÉRARD B. 1995 - Etude techno-économique de l'industrie magdalénienne de la grotte du Cavalier à Molompize (Cantal). Mémoire de maîtrise de l'université de Paris I, 82 p., dact.

BORDES F. 1958 - Nouvelles fouilles à Laugerie-Haute-Est, premiers résultats. L'Anthropologie, 62, p. 205-244.

BORDES F. 1978 - Le Protomagdalénien de Laugerie-Haute-Est (fouilles F. Bordes). Bulletin de la Société Préhistorique Française, 75, 11-12, p. 501-521.

BOSSELIN B. 1992 - Les industries lithiques du Protomagdalénien à partir des données du site du Blot (Cerzat, Haute-Loire). Thèse de doctorat de l'université de Franche-Comté, 3 t., 526 p., dact.

BOSSELIN B. 1997 - Le Protomagdalénien du Blot. Liège, ERAUL 64, 321 p.

BRÉZILLON M. 1971 - Les Tarterêts II, site paléolithique de plein-air à Corbeil-Essonnes (Essonne). Gallia-Préhistoire, XIV, 1, p. 3-40.

CHEYNIER A. 1957 - Les Gros-Monts à Nemours (Seine-et-Marne). Congrès Préhistorique de France, 1957, 22 p., 9 fig., 1 pl. 
CLAY R.-B. 1995 - Le Protomagdalénien de l'abri Pataud, niveau 2. In : BRICKER (dir.) Le Paléolithique supérieur de l'abri Pataud (Dordogne), les fouilles de H.L. Movius. Documents d'Archéologie Française, Paris, éditions de la Maison des Sciences de l'Homme, p. 67-87.

DELPORTE H. 1976 - Les civilisations du Paléolithique supérieur en Auvergne. La Préhistoire française, t. 1, vol. 2 , Paris, éditions du C.N.R.S., p. 1297-1304.

DEMARS P.-Y. 1994 - L'économie du silex au Paléolithique supérieur dans le nord de l'Aquitaine. Thèse de doctorat d'État de l'université de Bordeaux 1, 2 t., 549 et 270 p., dact.

DJINDJIAN F., KOSLOWSKI J. et OTTE M. 1999 - Le Paléolithique supérieur en Europe. Paris, Armand Colin, $474 \mathrm{p}$.

FLORÈs L. 1998 - Analyse technologique de l'industrie lithique du site des Tarterêts I, à Corbeil-Essonne (Essonne). Mémoire de maîtrise de l'université de Paris 1, dact.

GUICHARD J. et G. et MORALA A. 1989 - Rémanence de la technique Levallois au Paléolithique supérieur ancien. Deux sites du Bergeracois : Canaule 1 et Troche (Creysse). Documents d'Archéologie Périgourdine, Périgueux, t. 4, p. 5-20.

INIZAN M.-L., REDURON M., ROCHE H. et TIXIER J. 1995 - Technologie de la pierre taillée. Préhistoire de la pierre taillée $\mathrm{n}^{\circ} 4$, Meudon, CREP, $199 \mathrm{p}$.

KARLIN C. 1972 - Le débitage. In Leroi-Gourhan A. et Brézillon M. : Les fouilles de Pincevent. Essai d'analyse ethnographique d'un habitat magdalénien. VII ${ }^{\mathrm{e}}$ supplément à Gallia-Préhistoire, Paris, éditions du C.N.R.S., p. 263-277.

KARLIN C. 1975 - Le gisement paléolithique supérieur des Tarterêts I à Corbeil-Essonnes. II : le débitage. Gallia-Préhistoire, 18, 2, Paris, éditions du C.N.R.S., p. 341-357.

KLARIC L. 1999 - Un schéma de production lamellaire original dans l'industrie gravettienne de l'ensemble moyen du gisement du Blot à Cerzat (Haute-Loire). Mémoire de DEA de l'université de Paris 1, 2 vol, dact.

KONG-CHO S.-J. 1997 - Etude de l'industrie lithique de la couche 2 dite protomagdalénienne de l'abri Pataud, Les Eyzies, Dordogne : étude technologique et typologique. Thèse de doctorat du Muséum national d'Histoire naturelle, Institut de Paléontologie humaine, 374 p., ill.

LEROI-GOURHAN A. 1988 - Dictionnaire de la préhistoire. Paris, Presses universitaires de France, 1222 p. LORIN Y. 1996 - Rabier (commune de Lanquais, Dordogne), un site de plein air du Bergeracois. Mémoire de maîtrise de l'université de Paris 1,67 p.

MORALA A. 1990 - L'atelier périgordien supérieur de Rabier (Lanquais, Dordogne) : recherches sur l'origine des occupants du site sur les bases de la lithologie. Actes du Vème colloque international sur le silex, Cahiers du quaternaire $n^{\circ} 17$, vol. 2, p. 391-404.

NESPOULET R. 1996 - Le Périgordien VI de l'abri Pataud, Les Eyzies-de-Tayac, Dordogne. Étude technologique et typologique de l'industrie lithique de la couche 3. Thèse de doctorat, Muséum national d'histoire naturelle, Paris, $260 \mathrm{p}$.

oLIVE M. 1988 - Une habitation magdalénienne d'Étiolles. L'unité P15. Mémoires de la Société Préhistorique Française, t. 20, Paris, éditions de la Société Préhistorique Française, 175 p.

PELEGRIN J. 1992 - Quelques remarques sur le débitage. In Schmider B. : Marsangy, un campement des derniers chasseurs magdaléniens sur les bords de l'Yonne. ERAUL 55, Liège, p. 108-115.

PEYRONY D. et E. 1938 - Laugerie-Haute. Archives de l'IPH, Paris, mémoire 19, 81 p. 
PIGEOT M. 1987 - Magdaléniens d'Étiolles. Économie du débitage et organisation sociale unité d'habitation U5. XXVe supplément à Gallia-Préhistoire, Paris, éd. du C.N.R.S., 168 p.

PIGEOT N. 2004 (dir.) - Les derniers magdaléniens d'Étiolles. Perspectives culturelles et paléohistoriques. XXXVII ${ }^{\mathrm{e}}$ supplément à Gallia-Préhistoire, Paris, éd. du C.N.R.S., 351 p.

PLoux S. 1983 - Études de débitages expérimentaux : la marque du tailleur. In Mazière G., Minzoni-Alessio A. et Ploux S. - Préhistoire et technologie, cahier $n^{\circ} 2$. Publications de l'Unité de Recherches 28, Paris, éd. du C.N.R.S., p. 109-179.

SCHMIDER B. 1975 - Le gisement paléolithique supérieur des Tarterêts I, à Corbeil-Essonne (Essonne). Gallia-Préhistoire, t. XVIII, fasc. 2, p. 315-340.

SÉARA F. 1998 - Le gisement de Manhès-Ouest (commune de Saint-Mamet-la-Salvetat, Cantal). Service régional de l'archéologie d'Auvergne, Clermont-Ferrand, dact.

SONNEVILLE-BORDES D. de 1960 - Le Paléolithique supérieur en Périgord. Bordeaux, Delmas, 560 p.

SURMELY F. 1998 - Le peuplement de la moyenne montagne auvergnate, des origines à la fin du Mésolithique. Thèse de doctorat de l'université de Bordeaux 1. 2 t., 239 et 205 p., dact.

SURMELY F., CoStAMAGNo S. et HAYS M. Soumis - Le Gravettien et le Protomagdalénien en Auvergne. In RIGAUD J.-Ph. (dir.) - Entités régionales d'une paléoculture européenne : le Gravettien. Actes de la table ronde internationale des Eyzies-de-Tayac, juillet 2004.

SURMELY F. et MURAT R. 2003 - Études comparatives sur la résistance mécanique de divers silex lacustres et marins français. Actes de la table ronde internationale d'Aurillac « les matières premières lithiques en préhistoire ». Supplément à Préhistoire du Sud-Ouest, p. 337-342.

SURMELY F. et PASTY J.-F. 2003 - Les importations de silex en Auvergne durant la préhistoire. Actes de la Table ronde internationale d'Aurillac «Les matières premières lithiques en Préhistoire ». Supplément à Préhistoire du Sud-Ouest, p. 327-336.

SURMELY F., PASTY J.-P., ALIX Ph., MURAT R. et LIABEUF R. 2002 - Le gisement magdalénien du Pont-deLongues (Les-Martres-de-Veyre, Puy-de-Dôme). Bulletin de la Société Préhistorique Française, t. 99, $\mathrm{n}^{\circ} 1$, p. 13-38.

VALENTIN B., BODU P., HANTAÏ A. et PHILIPPE M. 1999 - L'industrie lithique (Marolles-sur-Seine). In : JULIEN M. et RIEU J.-L. (dir.) - Occupations du Paléolithique supérieur dans le sud-est du Bassin Parisien. Documents d'Archéologie française, 78, Paris, éd. de la Maison des Sciences de l'Homme, p. 82-97.

VALENTIN B. 1995 - Les groupes humains et leurs traditions au Tardiglaciaire dans le Bassin Parisien. Apports de la technologie lithique comparée. Thèse de doctorat de l'université de Paris 1, 2 t., 448 et 794 p., dact.

VIRMONT J. 1981 - Le bassin de l'Allier au Paléolithique supérieur. Industries et phases de peuplement. Thèse de $3^{\mathrm{e}}$ cycle de l'université d'Aix-Marseille., 378 p., dact.

\section{NOTES}

1. A la demande de l'un des rapporteurs de cet article, nous sommes amenés à rappeler que l'appellation Protomagdalénien est ici employée comme synonyme de ce que certains ont appelé aussi Périgordien VII (Bordes 1978) ou Gravettien final (Djindjian et al. 1999). Cette préférence s'explique par l'antériorité du terme (dû à D. Peyrony), par son emploi courant dans la littérature traitant des sites concernés (Laugerie-Haute, Pataud, Le Blot, Les Peyrugues) (Bosselin 1992), 
mais aussi par notre sentiment de l'individualité du Protomagdalénien par rapport au Gravettien, idée développée dans une étude sous presse (Surmely et al. sous presse).

2. Des datations sont en cours de réalisation pour le gisement du Blot.

3. Coordonnée par H. Delporte $(\dagger)$ et J.-P. Daugas.

4. Une dizaine de «niveaux » rapportables au Protomagdalénien ont pu être identifiés sur le site à l'occasion de la fouille (niveaux 34 à 22, Virmont 1981). J.-P. Daugas (étude en cours) les regroupe en trois ensembles principaux. Notre étude n'a pas permis de constater de différences significatives sur le mobilier lithique entre les «niveaux " ou «ensembles", ce qui plaiderait dans le sens de leur faible dispersion dans le temps et de leur unité culturelle. Dans cet article, nous avons donc considéré le mobilier lithique protomagdalénien comme un tout, réservant les décomptes précis par niveaux ou ensembles à la publication monographique.

5. Mesures prises sur les seules séries du gisement du Blot.

6. L'objet a été examiné par A. Morala (Musée National de Préhistoire). Il s'agit d'un petit galet allongé en schiste, brisé, mais qui ne porte pas de traces évidentes de percussion. A. Morala écarte résolument l'idée d'un chasse-lame, en raison de la nature minérale de la pièce qui ne se prête pas au débitage par percussion indirecte, surtout avec des talons en éperon.

7. Pour les décomptes effectués sur le produit des fouilles Peyrony de la couche F de LaugerieHaute-Est, il faut prendre en considération un probable tri de l'industrie effectué en faveur des «beaux » supports laminaires (Demars 1994).

8. C'est sur ce site qu'ont été définis les éperons, par le Dr Cheynier (Cheynier 1957). Un éperon massif est d'ailleurs reproduit dans les planches de dessin. Pourtant, l'examen que nous avons fait des séries a révélé la présence presque exclusive d'éperons fins, avec une domination très nette des talons lisses abrasés.

9. Nous remercions bien sincèrement $M$. Olive pour nous avoir autorisés à examiner le mobilier en cours d'étude.

\section{RÉSUMÉS}

A l'occasion de l'étude des séries lithiques protomagdaléniennes du gisement du Blot (Cerzat, Haute-Loire), nous avons constaté l'importance des aménagements de talons en éperon, liés au débitage de grandes lames. Un examen partiel des niveaux contemporains de l'abri Pataud et de Laugerie-Haute-Est (Les-Eyzies-de-Tayac, Dordogne) a permis de constater le même phénomène. Cette note vise à présenter ces aménagements et à les comparer avec quelques autres séries du Paléolithique supérieur et notamment du Magdalénien.

While studying some protomagdalenian lithic series from Le Blot site (Cerzat, Haute-Loire, France), we noticed that over $40 \%$ of the butts had been prepared with spurs, all of them related to the detachment of long blades. A quick study of the other protomagdalenian levels of the Pataud and Laugerie-Haute Est sites (Les-Eyzies-de-Tayac, Dordogne, France) shows the same thing.Through this note we mean to describe these preparations and to compare them with some other Upper paleolithic series, more particularly magdalenian sites. 
INDEX

Mots-clés : éperon, lames, Paléolithique supérieur, Protomagdalénien

Keywords : blades, Protomagdalenian, spur, Upper palaeolithic

\section{AUTEURS}

\section{FRÉDÉRIC SURMELY}

Géolab, UMR 6042 du CNRS, Maison de la Recherche - 4, rue Ledru 63000 Clermont-Ferrand. frederic.surmely@club-internet.fr

\section{PHILIPPE ALIX}

INRAP Rhône-Alpes/Auvergne - 12, rue Maggiorini 63500 Bron. 